\title{
ESTUDIO PRELIMINAR SOBRE EL USO DE ESCENAS DE PELÍCULAS COMO HERRAMIENTA EN LA FORMACIÓN DE TERAPEUTAS \\ PRELIMINARY STUDY ON THE USE OF MOVIE SCENES AS A TOOL IN THE TRAINING OF THERAPISTS
}

\author{
David Moreno-Mora \\ Jesús GARCÍA-MARTÍNEZ ${ }^{1}$ \\ Universidad de Sevilla \\ ${ }^{1}$ jgm@us.es
}

Cómo referenciar este artículo/How to reference this article:

Moreno-Mora, D. y García-Martínez, J. (2012). Estudio preliminar sobre el uso de escenas de películas como herramienta en la formación de terapeutas. Acción Psicológica, 9(2), 117-128 doi: http://dx.doi. org/10.5944/ap.9.2.4110

\section{Resumen}

El objetivo del presente trabajo es estudiar el uso de escenas de películas como herramienta en la formación de terapeutas. Para ello se tomó una muestra de 26 estudiantes de Psicología matriculados en la asignatura «Psicoterapias». Se dividió a los sujetos en dos grupos, uno de ellos visionó escenas de películas potencialmente terapéuticas y el otro grupo visionó escenas inocuas. Después del visionado se midió el efecto de las escenas en los sujetos mediante la aplicación del «Grupo de Transacción Interpersonal» GTI (Neimeyer, 1988), técnica de trabajo grupal en la que primero se mantienen conversaciones por parejas y después con todo el grupo. Los resultados mostraron que los sujetos del grupo de exposición terapéutica emitieron mayor número de respuestas de escucha y actos terapéuticos.

Palabras clave: formación de terapeutas, películas, grupo de transacción interpersonal.

\begin{abstract}
The aim this work is to study the use of scenes of movies as tool in therapists' training. For it there took a sample of 26 students of Psychology registered in the subject «Psychotherapies». It divided to the subjects in two groups, one of them viewed potentially therapeutic scenes of fims and another group viewed innocuous scenes. After the viewed, the effect of the scenes in the subjects was measured by the application of the «Interpersonal Transaction Group» ITG (Neimeyer, 1988), Technology of work with groups in that first conversations are kept in pairs and later with the whole group. The results showed that the subjects of the group of therapeutic exhibition emitted major number of answers of scout and therapeutic acts.
\end{abstract}

Keywords therapist training, films, interpersonal transaction group. 


\section{Introducción}

El entrenamiento de los terapeutas ha sido una cuestión extensamente estudiada en los últimos tiempos, dada su influencia en el curso de la terapia, la alianza terapéutica, y en el uso de determinadas técnicas. Para Safran y Muran (2005), dada la complejidad de las destrezas terapéuticas requeridas para el manejo de los procesos terapéuticos negativos es crítico que su entrenamiento sea de naturaleza más experiencial y subraye la auto-exploración como el principal vehículo de aprendizaje, ya que la adquisición de estas destrezas se ve mediada por factores personales y emocionales complejos. Así mismo, la destreza terapéutica incluye importantes aspectos intuitivos y creativos que son difíciles, si no imposibles, de enseñar. La investigación relativa a la naturaleza de la maestría profesional demuestra que los profesionales muy experimentados en diversos campos responden a situaciones relevantes de un modo flexible, creativo y sensible al contexto. A diferencia de los principiantes, que tienden a aplicar las reglas a modo de recetas de «libro de cocina».

Baker y Neimeyer (2003) realizaron un estudio en dos tipos de terapia grupal para la depresión (cognitivo-conductual y de autoayuda). En cada uno de ellos se diferenció entre clientes externalizadores e internalizadores. También se diferenciaron dos tipos de terapeuta, por un lado los que habían recibido un entrenamiento profesional y por otro los para-profesionales. Los resultados mostraron que los clientes externalizadores se veían más beneficiados por los para-profesionales, mientras los internalizadores obtenían mejores resultados con los terapeutas profesionalmente entrenados.

En otra investigación, Hinselroth, Ackerman, Clemence, Strassle y Handler (2002) estudiaron los efectos de un entrenamiento clínico estructurado (structured clinician training, SCT) en la alianza terapéutica. Para ello aplicaron dos tipos de tratamiento a dos muestras de 34 sujetos. Un primer grupo recibió tratamiento de terapeutas formados en un SCT y el otro grupo fue tratado usando un modelo de tratamiento típico no estructurado de trata- miento psicodinámico ecléctico. Los dos grupos obtuvieron buenas puntuaciones en alianza terapéutica general, pero el grupo SCT tuvo diferencias significativas en facetas de la alianza terapéutica (metas, tareas y vínculos).

Hersoug, Hoglend, Monsen y Havik (2001) encontraron que el entrenamiento y la formación de los terapeutas eran variables que estaban relacionadas positivamente con la alianza terapéutica cuando esta era evaluada por los terapeutas, pero no cuando la alianza era evaluada por el paciente.

Los resultados de este tipo de investigaciones justifican el estudio de factores que puedan servir como herramienta en la formación y entrenamiento de futuros terapeutas. Por ejemplo, las respuestas terapéuticas de escucha: Clarificación, paráfrasis, reflejo y síntesis; y otros aspectos centrales de la terapia en los que se debe incidir en la formación de futuros terapeutas (empatía, actitud genuina, aceptación positiva, etc.).

La herramienta propuesta en el presente estudio es el uso de escenas de películas complementada con el grupo de transacción interpersonal (GTI).

El uso de películas como herramienta adjunta dentro de una terapia narrativa ha ido creciendo durante los últimos años. Autores como Hesley y Hesley (1998), argumentan que las películas pueden ayudar a tratamientos pensados para ofrecer esperanza y aliento, reformular problemas, proporcionar modelos a seguir, identificar y reforzar fuerzas internas, potenciar la emoción, mejorar la comunicación, y ayudar a los clientes en su escala de valores. Berg-Cross, Jennings y Baruch (1990) mantienen que el cine ayuda a crear alianza terapéutica «por crear un puente común de entendimiento entre la angustia del cliente y la empatía del terapeuta». Informan que las películas dan a los clientes profundos insights sobre sus dilemas vitales, un profundo conocimiento de su personalidad, y crean unas metáforas terapéuticamente significativas, capturando la esencia del problema. Estos autores también ofrecen 4 sugerencias adicionales para el uso de escenas de películas: (a) el cliente debe estar activa y conscientemente trabajando en un dominio es- 
pecífico; (b) el terapeuta debe querer profundizar o ensanchar el dominio en el que el cliente está trabajando; (c) el terapeuta debe preparar adecuadamente al cliente para el film; y (d) el terapeuta debe procesar el video con el cliente poco después de ser visionado.

Mangín (1999) afirma que si las películas tienen la potencialidad de generar conductas negativas en las personas (violencia, etc.) lógicamente su buen uso tendrá la potencialidad de promover conductas positivas. Para este autor, el impacto de las películas es más en un nivel emocional que intelectual, ayudando a reducir la represión y otros mecanismos de defensa, además tiene la ventaja de permitir tratar de forma indirecta material que es difícil abordar de forma directa, para lo cual es preferible trabajar a un nivel más metafórico que literal.

El uso clínico de películas ha tenido diversas aplicaciones. Powell, Newgent, y Lee (2006) utilizaron el visionado guiado de una película, como ejemplo psicoeducativo, para aumentar la autoestima de adolescentes con problemas emocionales que participaban en un grupo de habilidades de afrontamiento; Garry, McDonald, Rey y Rosen (2002) evaluaron si la visión de determinadas películas, influía en las actitudes de estudiantes de medicina hacia la terapia electroconvulsiva; y Gelkopf, Gonen, Kurs, Melamed y Bleich (2006) estudiaron el efecto de películas cómicas en pacientes de esquizofrenia.

García-Martínez, Domínguez-Urquiza y Martínez-Andreu (2000) realizaron un estudio razonado del contenido de películas enteras o fragmentos de ellas buscando la adecuación para algunos hechos de la terapia narrativa. En dicho estudio muestran una lista de hechos en terapia narrativa y dan algunos ejemplos de películas y escenas que podrían ser adecuadas para ilustrar estos hechos.

Por otro lado, el GTI, procedimiento utilizado para explorar el efecto de las escenas de películas, es una técnica de trabajo grupal que busca inducir nuevos significados a través de la revisión de la experiencia propia y de las experiencias aportadas por los otros componentes del grupo, es decir, se trata de un aprendizaje de tipo colaborativo. Esta técnica se ha aplicado al trabajo con mujeres maltratadas (Neimeyer, 1988) y al trabajo con jóvenes delincuentes que ya están institucionalizados (Viney y Henry, 2002; Viney, Henry y Campbell, 2001) y a la reducción de conductas de riesgo (consumo de alcohol, sexo no protegido, riesgo de conducta antisocial) en adolescentes (Garcia-Martínez, Domínguez-Urquiza, Guerrero-Gómez, Morales-Hidalgo, PicóJiménez y Núñez-Remesal, 2004; Viney, Truneckova, Weekes y Oades, 1997). Los grupos de transacción interpersonal suelen funcionar de esta manera: 1) el grupo se inicia con un trabajo diádico en el que todos los participantes hablan por parejas con todos y cada uno de los miembros del grupo sobre un tema a trabajar en la sesión; 2) Trabajo de todo el grupo en sesión conjunta sobre ese mismo tema usando como material el obtenido en la rueda de díadas.

El objetivo del presente trabajo es estudiar si el visionado de escenas de películas facilita la aparición de respuestas de escucha y actos terapéuticos en terapeutas noveles durante un GTI posterior.

\section{Método}

\section{Participantes}

Alumnos de la facultad de Psicología de la Universidad de Sevilla (22 mujeres y 4 hombres), de entre 21 y 30 años de edad (Edad Media $=22.42 ; \mathrm{Sd}=2.14)$. Los sujetos eran voluntarios, se les reclutó durante las clases de la asignatura psicoterapias, que todos ellos tenían recién cursada en el momento de la evaluación. Psicoterapias es una asignatura optativa del primer cuatrimestre de $4^{\circ}$ curso en la que se imparten enseñanzas relacionadas con modelos terapéuticos de carácter fenomenológico, humanista y constructivista.

\section{Instrumentos de evaluación}

\section{Montajes de video}

Se utilizaron dos montajes de video elaborados por los investigadores: El montaje utilizado para la condición experimental constaba de escenas que potencialmente po- 
dían facilitar la emisión de respuestas de escucha y actos terapéuticos en los espectadores. El montaje empleado para el grupo control constaba de escenas inocuas respecto a la posible emisión de respuestas de escucha y actos terapéuticos. Estas escenas pertenecían a las mismas películas que las usadas en la condición experimental y fueron proyectadas en el mismo orden. Las escenas incluidas en ambos montajes pertenecen a las siguientes películas: Tomates verdes fritos (Avnet, 1991), Beautiful girls (Demme, 1996), Cosas que nunca te dije (Coixet, 1996), Criaturas celestiales (Jackson, 1994), Don Juan de Marco (Leven, 1995), El club de los poetas muertos (Weir, 1989), Hombre mirando al sudeste (Subiela, 1986), El cartero y Pablo Neruda (Radford, 1994), Infernal Affairs (Lau y Mak, 2002), K-Pax (Softley, 2001), Mumford (Kasdan, 1999), No amarás (Kieslowski, 1988), Noche en la tierra (Jarmusch, 1991), Otra mujer (Allen, 1988), Pequeña Miss Sunshine (Dayton y Faris, 2006), El indomable Will Hunting (Van Sant, 1997).

A continuación se muestra dos ejemplos de escenas utilizadas en los montajes, una activa y otra inocua, ambas pertenecientes a la misma película:

\section{Escena activa}

Película: El cartero y Pablo Neruda (1994) M. Radford. Desde el minuto 21:00 hasta el 23:32.

Descripción: Pablo Neruda explica a su cartero como a través de las metáforas y la poesía puede aprender a expresar y entender sus propios sentimientos, enriqueciendo así su experiencia.

\section{Escena inocua}

Película: El cartero y Pablo Neruda (1994) M. Radford. Desde el minuto 11:23 hasta el 12:40.

Descripción: Pablo Neruda recibe el correo, gasta una broma a su mujer y da una propina al cartero.

\section{Tarjetas de emoción}

Las sesiones de GTI utilizan un criterio de medida del efecto de las sesiones grupales conocido como tarjetas de emoción (Landfield y Rivers, 1975; Neimeyer, 1988; Garcia-Martínez, Guerrero-Gómez, Picó-Jiménez y Morales-Hidalgo, 2005) y, que no son más que hojas en las que los participantes anotan pensamientos, emociones y otros tipos de significados que les evocan lo ocurrido en las sesiones. El sistema de categorías utilizado para la sesión de GTI constó de las siguientes respuestas de escucha (Cormier y Cormier, 2000) y actos terapéuticos:

Respuestas de escucha:

- Clarificación: está destinada a averiguar lo qué quiere decir el cliente. Sus objetivos específicos son obtener más información y comprobar la que ya se tiene. Se hace de forma interrogativa.

- Paráfrasis: repetición del contenido del mensaje, pero trasladándolo al lenguaje del terapeuta. Se comunica siempre como una afirmación.

- Reflejo: es la respuesta utilizada para remarcar la parte emocional del mensaje traduciéndola a términos del terapeuta.

- Síntesis: Intervención que agrupa dos o más reflejos, paráfrasis o ambas.

Los otros elementos centrales de la terapia considerados son:

- Referencias a la empatía: Esta medida de empatía consiste en las referencias a la empatía como acto terapéutico (capacidad de experimentar las cosas desde el punto de vista del cliente, pero sin confundirse con este).

- Actitud genuina: consiste en transmitir en todo momento una actitud personal, no forzada, por parte del terapeuta, de modo que se haga evidente que es la persona del terapeuta quién está en la situación de terapia y no se está desempeñando un mero rol. 
- Aceptación positiva: es la demostración de respeto, interés y valoración de la persona del cliente. Implica compromiso, esfuerzo por entender al paciente, tener una actitud no valorativa, dar protección y cercanía, y el uso de la inmediatez.

- Referencias al yo: Relacionado con la actitud genuina y la autorrevelación (dar información de tipo personal). También relacionado con la inmediatez.

- Referencias a proporcionar ayuda: Relacionado con la aceptación positiva (protección y cercanía).

- Referencias a preocupación por los otros: Relacionado con la empatía y la aceptación positiva (Compromiso, esfuerzo por entender).

- Referencias a técnicas terapéuticas concretas: Relacionado con la aceptación positiva y la creatividad.

\section{Procedimiento}

Se dividió aleatoriamente a los sujetos en dos grupos, los del grupo de exposición terapéutica visionaron el montaje activo y los del grupo control visionaron el montaje inocuo. La sesiones se realizaron en días diferentes, siendo el montaje de video la única diferencia entre una y otra. Las sesiones empezaron con el visionado del correspondiente montaje de video, posteriormente los sujetos escribieron sus impresiones sobre las escenas vistas en tarjetas de emoción. Después se realizaron debates por parejas de unos 4 minutos cada uno, de forma que cada sujeto conversaba con otros 5 participantes que le eran asignados aleatoriamente. Después se realizó un debate en común en el que los sujetos intercambiaron sus impresiones finales sobre la sesión. El contenido de los tipos de debate también fue escrito en tarjetas destinadas a ello.

\section{Resultados}

Se realizó una regresión lineal con pruebas de efecto intra e intersujetos con el paquete estadístico SPSS 14 para estudiar el efecto que producía en los sujetos el ser expuestos a diferentes montajes de video y si este efecto variaba a lo largo del GTI.

A continuación se presenta una tabla con las variables en las que el número de respuestas fue mayor en el grupo de exposición terapéutica. A, B y C se corresponden con las tres tarjetas de emoción administradas tras cada momento del GTI:

Tabla 1

Frecuencia de respuestas de escucha y actos terapéuticos durante el GTI en la muestra terapéutica y en la inerte

\begin{tabular}{|c|c|c|c|c|c|c|c|c|c|c|c|c|c|c|}
\hline \multirow{3}{*}{$\begin{array}{c}\text { Exposición } \\
\text { Variable }\end{array}$} & \multicolumn{6}{|c|}{ Media } & \multicolumn{6}{|c|}{ SD } & \multirow{3}{*}{$F$} & \multirow{3}{*}{$\mathbf{P}$} \\
\hline & \multicolumn{3}{|c|}{ Inerte } & \multicolumn{3}{|c|}{ Terapéutica } & \multicolumn{3}{|c|}{ Inerte } & \multicolumn{3}{|c|}{ Terapéutica } & & \\
\hline & A & B & C & A & B & C & A & B & C & A & B & C & & \\
\hline Reflejo & .17 & .17 & .00 & 2.00 & .64 & .00 & .39 & .39 & .00 & 1.74 & .93 & .00 & 13.81 & .001 \\
\hline Síntesis & .50 & .80 & .00 & 1.14 & .36 & .36 & .80 & .29 & .00 & 1.56 & .50 & .50 & 4.60 & .042 \\
\hline Acept. Posit. & .00 & .00 & .00 & .50 & .29 & .29 & .00 & .00 & .00 & .76 & .47 & .83 & 10.51 & .003 \\
\hline Actitud Gen & .80 & .00 & .00 & .43 & .36 & .21 & .29 & .00 & .00 & .65 & .63 & .43 & 6.88 & .015 \\
\hline Capacitación & .42 & .00 & .17 & .86 & .64 & 1.00 & .79 & .00 & .39 & .77 & .93 & 1.04 & 14.82 & .001 \\
\hline Prop. ayuda & .33 & .00 & .08 & 2.50 & .64 & .57 & .65 & .00 & .29 & 1.79 & .75 & .85 & 24.88 & .000 \\
\hline Interés otros & .08 & .00 & .00 & .50 & .07 & .14 & .29 & .00 & .00 & .65 & .27 & .36 & 7.94 & .010 \\
\hline Ref. técnicas & .33 & .00 & .08 & 1.00 & .29 & .43 & .65 & .00 & .29 & 1.11 & .47 & .76 & 5.04 & .034 \\
\hline
\end{tabular}


En la tabla 2 se muestran las tendencias de algunas de la variables durante los tres momentos del GTI (A, B y C).

En el Apéndice se presentan las gráficas con los resultados para cada variable dependiente. Los puntos 1, 2 y 3 de las figuras del 1 al 6 responden a las respuestas en las tarjetas A, B y C del GTI. El grupo criterio está representado en color gris claro y el control en gris oscuro.

\section{Discusión}

El primer objetivo planteado era comprobar si los terapeutas que ven escenas potencialmente terapéuticas emitían más respuestas de escucha y actos terapéuticos que los que veían escenas inocuas. Los resultados muestran cómo las respuestas de escucha de reflejo $(p=.001)$ y síntesis $(p=.042)$ tienen una frecuencia significativamente mayor en el grupo de exposición terapéutica. Esto indica que los sujetos que vieron escenas significativas identificaron contenidos emocionales en el mensaje traduciéndolos a sus propios términos, y que fueron capaces de captar temas recurrentes a lo largo del montaje visionado.
También aparecen de forma significativamente mayor en el grupo experimental, otros elementos centrales de la terapia como son la actitud genuina $(p=.015)$, la aceptación positiva $(p=.003)$, referencias a la capacitación $(p=.001)$, referencias a proporcionar ayuda $(p=.000)$, referencias al interés por los otros $(p=.01)$ y referencias a técnicas terapéuticas concretas $(p=.034)$. La aparición de todos estas respuestas de escucha y actos terapéuticos durante el desarrollo del GTI en el grupo de exposición terapéutica no hace más que confirmar que los terapeutas en formación vivieron la experiencia como algo similar a la terapia, como un ensayo o una práctica, sobre todo teniendo en cuenta que todas estas respuestas de escucha y actos terapéuticos deben ser tenidas en cuenta en la formación de futuros terapeutas debido a su importancia en el desarrollo de una buena relación terapéutica. Otro aspecto a destacar es que los dos tipos de montaje resultaron ser significativamente diferentes entre si, ya que, el que se pretendía que fuese terapéutico realmente lo fue, si bien es verdad que no todos los clips tuvieron la misma importancia para los sujetos; del mismo modo, el montaje del grupo control resultó realmente inocuo.

Tabla 2

Tendencia de las respuestas de escucha y actos terapéuticos durante el GTI en la muestra terapéutica y en la inerte

\begin{tabular}{|c|c|c|c|c|c|c|c|c|c|c|c|c|c|c|c|}
\hline \multirow{3}{*}{$\begin{array}{c}\text { Exposición } \\
\text { Variable }\end{array}$} & \multicolumn{6}{|c|}{ Media } & \multicolumn{6}{|c|}{ SD } & \multirow[b]{3}{*}{$\mathbf{F}$} & \multirow[b]{3}{*}{ Tendencia } & \multirow[b]{3}{*}{ Sig. } \\
\hline & \multicolumn{3}{|c|}{ Inerte } & \multicolumn{3}{|c|}{ Terapéutica } & \multicolumn{3}{|c|}{ Inerte } & \multicolumn{3}{|c|}{ Terapéutica } & & & \\
\hline & A & B & C & A & B & C & A & B & C & A & B & C & & & \\
\hline Paráfrasis & 1.50 & 1.08 & .42 & 2.79 & .86 & .71 & 1.83 & 1.08 & .67 & 2.55 & .86 & 1.14 & 10.88 & Lineal & .003 \\
\hline Reflejo & .17 & .17 & .00 & 2.00 & .64 & .00 & .39 & .39 & .00 & 1.74 & .93 & .00 & 12.51 & Lineal & .002 \\
\hline Síntesis & .50 & .80 & .00 & 1.14 & .36 & .36 & .80 & .29 & .00 & 1.56 & .50 & .50 & 6.86 & Lineal & .015 \\
\hline Ref. empatía & .50 & 1.83 & .83 & .71 & 1.71 & .64 & .80 & 1.03 & 1.12 & .91 & .83 & .84 & 21.87 & Cuadrática & .000 \\
\hline Ref. al yo & 5.00 & 3.17 & 2.58 & 6.00 & 3.79 & 2.71 & 2.45 & 1.53 & 1.31 & 2.91 & 1.19 & 1.07 & 45.94 & Lineal & .000 \\
\hline Prop. ayuda & .33 & .00 & .08 & 2.50 & .64 & .57 & .65 & .00 & .29 & 1.79 & .75 & .85 & 7.919 & Lineal & .000 \\
\hline Ref. técnicas & .33 & .00 & .08 & 1.00 & .29 & .43 & .65 & .00 & .29 & 1.11 & .47 & .76 & 5.396 & Cuadrática & .029 \\
\hline
\end{tabular}


También cabe destacar que algunas de las respuestas de escucha siguen tendencias diferentes en los tres momentos del GTI. Las respuestas de escucha de paráfrasis $(\mathrm{F}=10.188$, $p=.003)$ y síntesis $(\mathrm{F}=6.86, p=.15)$, así como las referencias al yo $(\mathrm{F}=45.94, p=.000)$ van reduciendo su frecuencia conforme avanza el GTI en los dos tipos de exposición. Llama la atención este último dato sobre todo teniendo en cuenta que el objetivo del GTI es un aprendizaje de tipo colaborativo en el que se busca inducir nuevos significados a través de la revisión de la experiencia propia y de las experiencias aportadas por los otros componentes del grupo. En la misma dirección, se puede hablar del ascenso de las referencias a la empatía $(\mathrm{F}=21.87, p=.000)$ en los dos grupos a partir del momento 2 del GTI, el de los debates por parejas, para luego volver a disminuir en el momento tres. Estos dos datos considerados conjuntamente, es decir, la disminución de las referencias al yo y el aumento de las referencias a la empatía conforme avanza el GTI, son los que hacen pensar que esta técnica podría también utilizarse en la formación de terapeutas de forma conjunta con los clips de películas. Dicho de otra manera, el sujeto pasa de centrarse en el si mismo (cuando los sujetos escriben en un primer momento lo que les ha sugerido el montaje) a considerar el punto de vista del otro (cuando el sujeto comparte su experiencia con otra persona).

Algunas respuestas de escucha siguen tendencias diferentes en uno y otro grupo. Así, se aprecia un descenso de las respuestas de clarificación $(\mathrm{F}=3.692, p=.33)$ y reflejo $(\mathrm{F}=8.115$, $p=.003)$ en el grupo de exposición terapéutica conforme avanza el GTI mientras que se mantienen constantes en unos niveles bajos en el grupo de exposición no terapéutica, lo cual es lógico si se considera que es en un primer momento cuando uno quiere clarificar las dudas que le genera el mensaje recibido, sobre todo si este es complejo, cosa que no ocurre cuando el mensaje no plantea ninguna interrogante ni presenta contenidos emocionales. La misma tendencia se encuentra con las referencias a proporcionar ayuda $(\mathrm{F}=6.489, p=.010)$.

Dicho de otro forma, el montaje terapéutico es adecuado para promover respuestas terapéuticas y puede ser útil para el entrenamiento en psicoterapia.

A la luz de estos resultados se considera pertinente realizar un estudio de mayor envergadura, ya que, a pesar de contar con una muestra de solo 26 sujetos los resultados son como mínimo alentadores. Por un lado se ha hallado que escenas de películas bien seleccionadas pueden elicitar respuestas de escucha y actos terapéuticos que también aparecen (y deben aparecer) en terapia, si bien es cierto que en futuros estudios habría que eliminar las escenas del montaje terapéutico que no resultaron significativas para los sujetos, así como incluir nuevas escenas que demuestren su potencial.

También se observa como el GTI, que fue la técnica de recogida de datos, puede servir en futuros estudios como técnica de formación de terapeutas incluyendo mayor número de sesiones, ya que en este estudio solo se realizó una, aunque fue suficiente para ver cómo los sujetos se iban enriqueciendo a partir de la experiencia de los demás durante su desarrollo. Por todo ello, se hace necesaria la realización de estudios con una muestra mayor, más heterogénea, y con mayor número de sesiones, con ello podríamos ver los efectos conjuntos del visionado de las escenas de cine y del GTI a lo largo de diferentes sesiones. También sería de interés comprobar las posibles mejoras de los terapeutas noveles en un ámbito aplicado de acción terapéutica y no solo en una simulación grupal, ya que el hecho de que aparezcan respuestas de escucha después del visionado de escenas de películas, no garantiza que vayan a aparecer en una situación real. 


\section{Referencias}

Allen, W (1988). Otra mujer [Another woman]. USA: Jack Rollins \& Charles H. Joffe Productions.

Avnet, J. (1991). Tomates verdes fritos. USA: Act III Communications.

Baker, K. D. y Neimeyer, R. A. (2003). Therapist Training and Client Characteristics as Predictors of Treatment Response to Group Therapy for Depression. Psychotherapy Research, 13, 135-151. doi:10.1093/ptr/kpg015

Berg-Cross, L., Jennings, P. y Baruch, R. (1990). Cinematherapy: Theory and application. Psychotherapy in Private Practice, 8, 135-156.

Coixet, I. (1996). Cosas que nunca te dije [Things I never told you]. España: Carbo Films.

Cormier, W. H. y Cormier, L. S. (2000). Estrategias de entrevista para terapeutas: habilidades básicas e intervenciones congnitivo-conductuales [Interview strategies for therapies: basics skills and cognitive behavioural interventions]. Bilbao, España: Desclée de Brouwer.

Dayton, J. y Faris, V. (2006). Pequeña Miss Sunshine [Little Miss Sunshine]. USA: Fox Searchlight Pictures.

Demme, T. (1996). Beautiful girls. USA: Miramax Films.

García-Martínez, J., Domínguez-Urquiza, J. M., Guerrero-Gómez, R., Morales-Hidalgo, F., Picó-Jiménez, A. y Núñez-Remesal, A. M. (2004). Personalidad y Riesgo de Presentar Conducta Antisocial: Efectos de un Programa Preventivo. Análisis y Modificación de Conducta, 134. 905-933.

García-Martínez, J., Domínguez-Urquiza, J. M. y Martínez Andreu, C. (2000, marzo). One Image Is Better Than a Thousand Words: Using Movies in Narrative Therapy. Póster presentado en el XXX Congress of European Association for Behavioural \& Cognitive Therapies. Granada.

García-Martínez, J., Rafael Guerrero-Gómez, R., Domínguez-Urquiza, J. M., Picó-Jiménez, A. y Morales Hidalgo, F. (2005, junio). En Session Changes During a Preventive Program for Antisocial Behaviour. 5th International Congress of Cognitive Psychotherapy \& 9th World Congress on Constructivism. Goteborg (Suecia).

Garry, W., McDonald, A., Rey, J. M. y Rosen, A. (2002). Medical Student Knowledge and Attitudes Regar- ding ECT Prior to and After Viewing ECT Scenes from Movies. The journal of ECT. 18(1), 43-46.

Gelkopf, M., Gonen, B., Kurs, R., Melamed, Y. y Bleich, A. (2006) The Effect of Humorous Movies on Inpatients With Chronic Schizophrenia. The Journal of Nervous and Mental Disease, 194 (11), 880-883.

Hersoug, A. G., Hoglend, P., Monsen, J. T. y Havik, O. E. (2001). Quality of working early alliance in psychotherapy: Therapist variables and patient/ therapist similarity as predictors. Journal of Psychotherapy Practice and Research, 10, 205-216.

Hesley, J. W. y Hesley, J. G. (2001). Rent two films and let's talk in the morning: Using popular movies in psychotherapy (2. ${ }^{\mathrm{a}}$ Ed.). New York: Wiley.

Hinselroth, M. J., Ackerman, S. J., Clemence, A. J., Strassle, C. G. y Handler, L. (2002). Effects of structured clinician training on patient and therapist perspectives of alliance early in psychotherapy. Psychotherapy: Theory, Research, Practice, Training, 39, 309-323.

Jackson, P. (1994). Criaturas celestiales [Heaven Creatures]. Nueva Zelanda: Fontana Productions.

Jarmusch, J. (1991). Noche en la Tierra [Night on Earth]. USA: Victor Company of Japan (JVC).

Kasdan, L. (1999). Mumford. USA: Touchstone Pictures.

Kieslowski, K. (1988). No amarás [A short film about love]. Polonia: Zespol Filmowy «Tor».

Landfield, A.W. y Rivers, P.C. (1975). An introducction to interpersonal transaction and rotating dyads. Psychotherapy: Theory, research and practice, 12(4), 365-373. doi:10.1037/h0086463

Leven, J. (1994). Don Juan de Marco. USA: New Line Cinema.

Mangin, D. (1999). Cinema therapy: How some shrinks are using movies to help their clients cope with life and just feel better. Health and Body, May 27. Recuperado de http://www.salon.com/health/ feature/1999/05/27/film_therapy/index.html/

Neimeyer, R. A. (1988). Clinical guidelines for conducting interpersonal transaction groups. International Journal of Personal Constructs Psychology, 1, 181-199. doi:10.1080/10720538808412775

Powell, M. L., Newgent, R. A. y Lee, S. M. (2006). Group cinematherapy: Using metaphor to enhance adolescent self-esteem. Arts in psychotherapy, 33, 247-253. doi:10.1016/j.aip.2006.03.004. 
Radford, M. (1994). El cartero y Pablo Neruda. Italia: Cecchi gori club tiger cinematográfica.

Safran, J. D. y Muran, J. C. (2005). La alianza terapéutica: Una guía para el tratamiento relacional [The Therapeutic Aliance: a guide for relational treatment]. Bilbao, España: Desclee de Brouwer.

Softley, I. (2001). K-Pax. USA: IMF internationale.

Subiela, E. (1986). Hombre mirando al sudeste [A man watching to Southwest]. Argentina: Cinequanon.

Van Sant, G. (1997). El indomable Will Hunting. [Good Will Hunting]. USA: Be Gentlemen Limited Partnership.

Viney, L. L. y Henry, R. M. (2002). Evaluating personal construct and psychodynamic group work with adolescent offenders and nonoffenders. En R. A. Neimeyer y G. J. Neimeyer,
Greg (Eds.). (2002), Advances in personal construct psychology new directions and perspectives (pp. 259-294). Westport, CT: Praeger Publishers/Greenwood.

Viney, L. L., Henry, R. M. y Campbell, J. (2001). The impact of group work on offender adolescents. Journal of Counseling and Development, 79, 373-381.

Viney, L. L., Truneckova, D., Weekes, P. y Oades, L. (1997). Personal construct group work with school-based adolescents: Reduction of risktaking. Journal of Constructivist Psychology, 10, 167-186. doi:10.1080/10720539708404620

Wai Keng, L. y Siu Fai, M. (2002). Juego sucio. [Mou gaan dou]. Hong Kong: Media Asia Films.

Weir, P. (1989). El club de lo poetas muertos. [Dead poets society]. USA: Touchstone Pictures. 


\section{APÉNDICE A}

Frecuencia y tendencia de las respuestas de escucha y actos terapéuticos durante el GTI en la muestra terapéutica y en la inerte

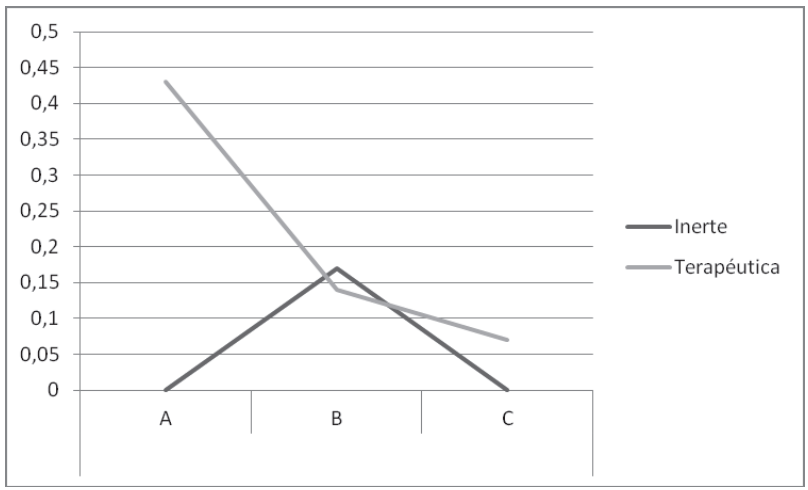

Figura 1. Clarificación.

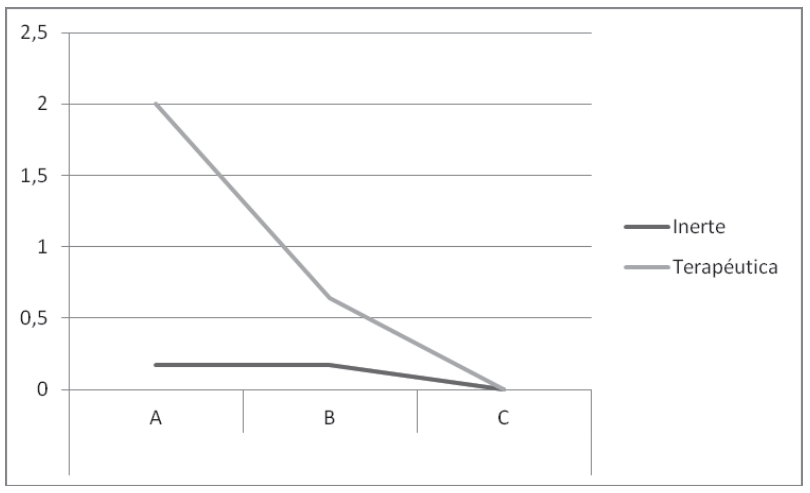

Figura 3. Reflejo.

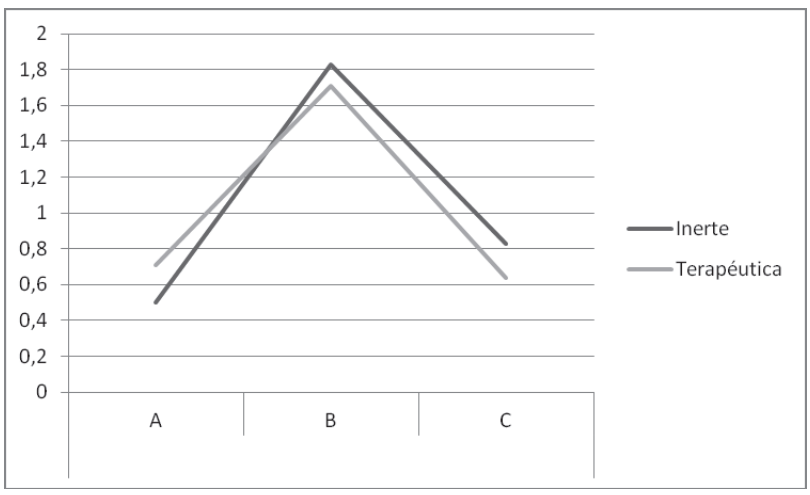

Figura 5. Referencias a la empatía.

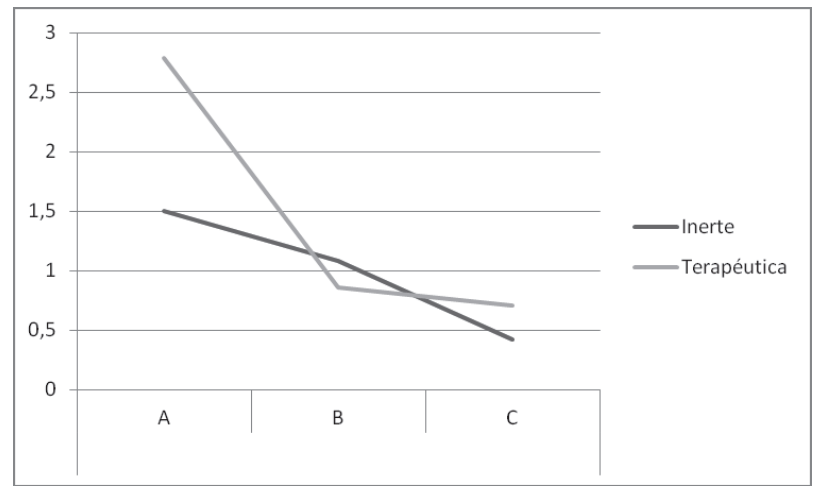

Figura 2. Paráfrasis.

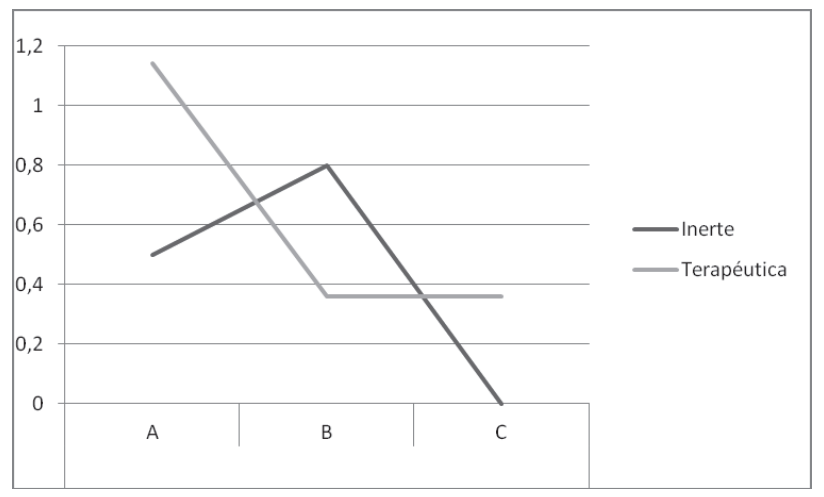

Figura 4. Síntesis.

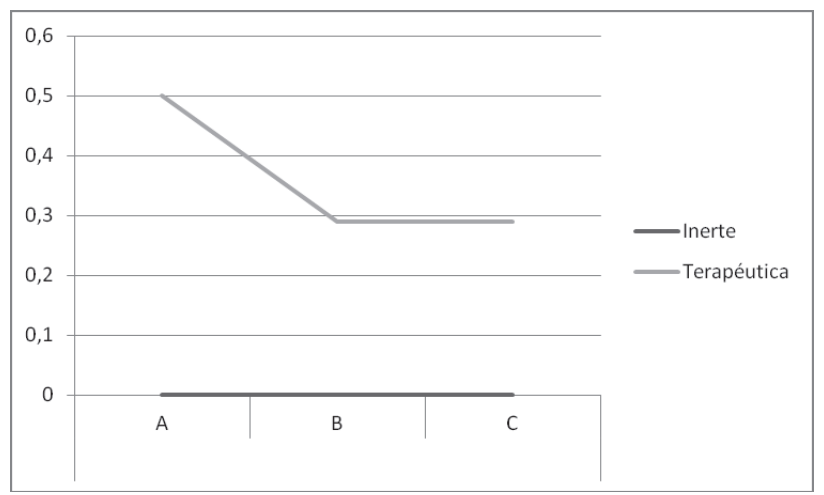

Figura 6. Aceptación positiva. 


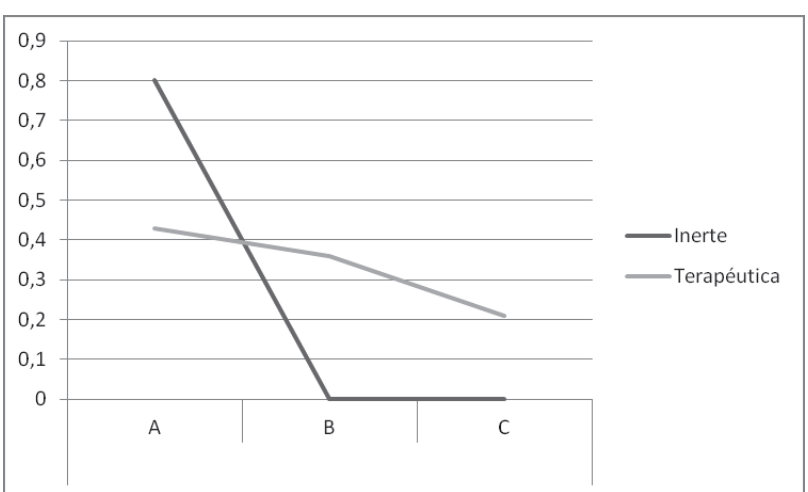

Figura 7. Actitud Genuina.

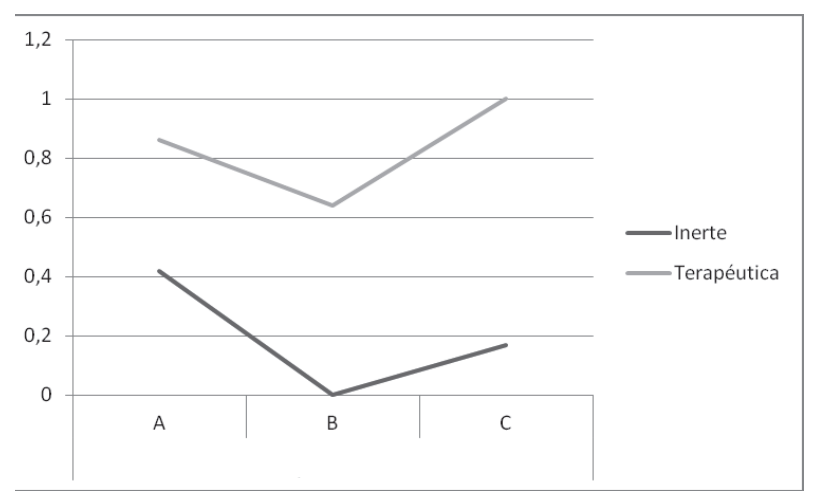

Figura 9. Capacitación.

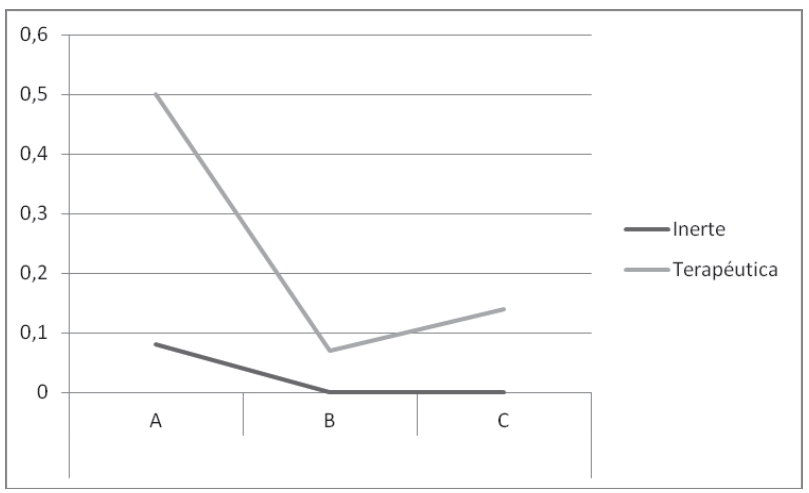

Figura 11. Interés en los otros.

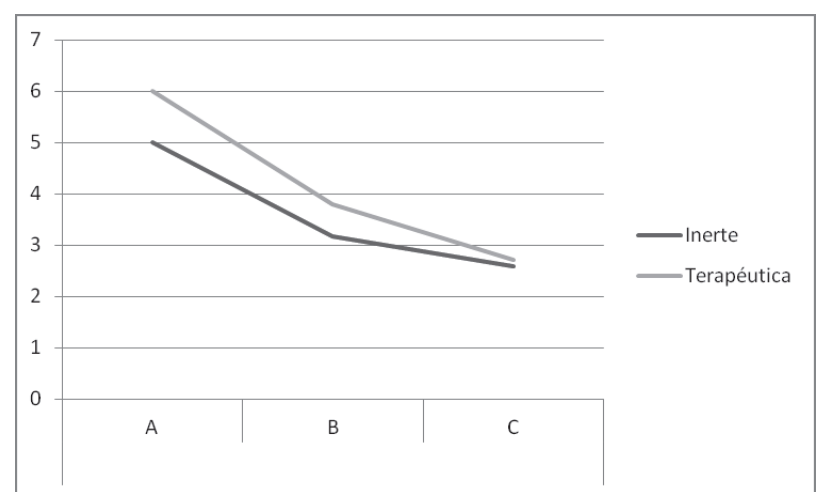

Figura 8. Referencias al yo.

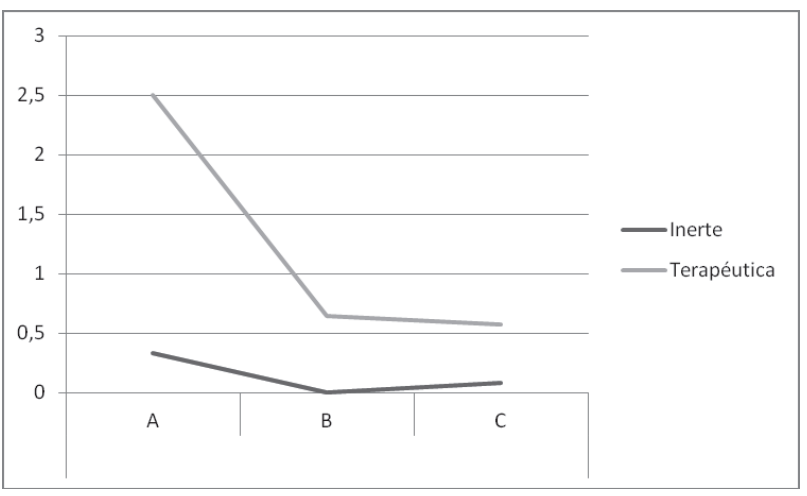

Figura 10. Proporcionar ayuda.

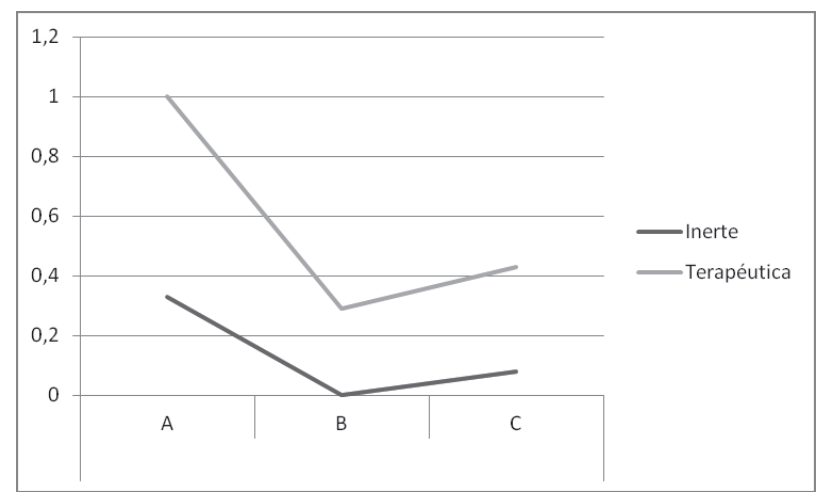

Figura 12. Referencia a técnicas. 
